\title{
Pancreatic Enzyme Supplementation Improves Quality of Life in Patients Following Surgery for Upper GI Cancer
}

\author{
Callum Alexander, Robert Kay, Sajid Waheed Rahman, Chris Deans*
}

*Corresponding author:

Chris Deans, MD

University Department of Surgery

Royal Infirmary

51 Little France Crescent

EH16 4SA, Edinburgh, UK

Tel 00441312423620

E-mail: chris.deans@nhslothian.scot.nhs.uk

Received: 27.02.2021

Accepted: 04.05.2021

Copyright @ Celsius Publishing House www.sgo-iasgo.com
University Department of Surgery, Royal Infirmary, 51 Little France Crescent, Edinburgh, UK, EH16 4SA

\section{ABSTRACT}

Background: Unpleasant abdominal symptoms are common following surgery for upper gastrointestinal (UGI) cancer and may occur secondary to pancreatic exocrine insufficiency (EPI). This study investigated symptoms of EPI in patients following surgery and assessed the effect of pancreatic enzyme supplementation (PERT) on these symptoms and the effect of supplementation on quality of life.

Methods: Patients were assessed for symptoms of EPI using a novel questionnaire. Patients who reported two or more symptoms suggestive of EPI were prescribed PERT. Abdominal symptoms were reassessed following treatment. Quality of life (QoL) was studied using the SF-36 questionnaire before and after treatment. Faecal elastase was measured in a patient subgroup.

Results: Fifty-six out of 57 patients (98\%) reported at least two symptoms of EPI. Following PERT every patient reported fewer abdominal symptoms; median 5 symptoms before treatment reduced to two symptoms following treatment $(p<0.0001$; Wilcoxon rank). Reduced faecal elastase concentration was associated with more frequent abdominal symptoms; median 5 symptoms versus 3 symptoms ( $p=0.043$; Mann Whitney $\mathrm{U}$ test). PERT increased quality of life scores for every patient in each of the 5 principle health domains.

Conclusion: Symptoms of EPI are common among patients following UGI cancer surgery. PERT reduces unpleasant abdominal symptoms and this leads to significant improvements in quality of life across global health domains. PERT should be offered to all post-operative UGI cancer patients with symptoms suggestive of EPI.

Key words: esophageal cancer; gastric cancer, exocrine pancreatic insufficiency, pancreatic enzyme replacement, PERT

\section{INTRODUCTION}

Surgical resection is the mainstay of treatment with curative intent for selected patients with esophageal and gastric cancer. Improvements in survival rates following surgery has led to an increased focus on health-related quality of life following surgery (1). There is an important need to address the effect of surgical treatment on post-operative symptoms and quality of life, in addition to cure rates, when considering survivorship. Unpleasant abdominal symptoms are common following surgery for upper gastrointestinal (UGI) cancer and are important determinants of quality of life as many of these symptoms may affect 
emotional, social and physical well-being. Symptoms such as abdominal cramps and diarrhoea are often regarded as inevitable consequences of surgery, resulting from changes to the anatomical orientation of the gastrointestinal tract and alterations to underlying gastrointestinal physiology. These symptoms are often attributed to 'dumping syndrome' and other conditions such as small intestine bacterial overgrowth (SIBO) which may lead to chronic maldigestion syndrome characterised by symptoms including abdominal cramping, diarrhoea, bloating, steatorrhoea and weight loss (2).

Exocrine pancreatic insufficiency (EPI) has also been reported in patients following surgery for oesophagogastric cancer and may contribute towards postoperative maldigestion syndrome $(1,3,4)$. Following esophagogastric resection neural signalling pathways from the esophagus/stomach to the pancreas are disrupted thus attenuating pancreatic exocrine function and activation $(5,6)$. Consequently there is reduced or uncoordinated release of pancreatic digestive enzymes resulting in an inability to effectively digest food. Clinically EPI mimics dumping syndrome and SIBO with abdominal cramping, diarrhoea, steatorrhoea and weight loss. As EPI and dumping syndrome/SIBO have significant symptomatic overlap it is therefore possible that some of the abdominal symptoms experienced following UGI surgery are attributable to EPI and would therefore improve with pancreatic enzyme replacement therapy (PERT).

PERT is an oral enzyme supplementation therapy taken with meals to mimic the enzymatic milieu created by the exocrine pancreas in normal health Creon ${ }^{\circledR}$ is a commercially available PERT containing lipases, proteases and amylases derived from the porcine pancreases catalysing the breakdown of fats, proteins and carbohydrates respectively. It is not systemically absorbed and has no contra-indications or known drug interactions making it a very safe management option (7). It is also very cheap making it a very cost-effective treatment.

The aim of this pilot study was to investigate symptoms of exocrine pancreatic insufficiency (EPI) in patients following surgery for UGI cancer and to assess the effect of pancreatic enzyme supplementation on abdominal symptoms and the effect of supplementation on patient-reported global quality of life.

\section{MATERIALS AND METHODS}

\section{Patient Selection}

All patients who have previously undergone surgery for carcinoma of the oesophagus or stomach were eligible for the study. Surgical procedures previously undertaken included Ivor Lewis esophagogastrectomy (including hybrid approach), thoracoscopic-assisted three-stage esophagectomy, subtotal gastrectomy and total gastrectomy with Roux-en-Y reconstruction. There were no limitations regarding time from surgery and enrolment to the study. As there was no control group or reliable historical data we decided to include all postoperative patients irrespective of the time interval from surgery. This was to investigate whether there was a time dependent effect on reported symptoms - ie did abdominal symptoms settle spontaneously with time following surgery. Patients who had surgery for benign disease or gastrointestinal tumours (GIST) were excluded. Patients who were receiving oncological treatments (chemotherapy or radiotherapy) at the time of the study were also excluded. Patients were recruited during attendance at routine outpatient clinic follow-up and all patients considered themselves 'well' at the time of recruitment. After appropriate written consent patients were issued with information leaflets about the study and letters were generated to the patient's General Practitioner, who also received an information sheet regarding the study. Patients were made aware that they were free to withdraw from the study at any time.

\section{Assessment of symptoms of exocrine pancreatic insufficiency}

There is no published validated patient questionnaire dedicated to symptoms for pancreatic insufficiency. We therefore devised our own questionnaire comprising ten symptoms of EPI commonly reported in the literature (810) (table 1). Patients were asked about the following symptoms that may suggest EPI: weight loss, greasy stools, steatorrhoea, watery stools, increased stool

Table 1 - Questionnaire designed to assess for symptoms of exocrine pancreatic insufficiency (EPI)

\begin{tabular}{l}
\hline Symptom \\
Have you lost weight recently? \\
Are yours stools particularly greasy or slimy? \\
Do your stools seem to float or been more difficult to flush? \\
Do your stools have a particularly bad odour? \\
Have you been passing bowel motions more frequently than usual? \\
Have your stools been more runny or watery? \\
Have you been passing more wind than usual? \\
Have you experienced abdominal cramps or discomfort recently? \\
Have you felt more bloated than usual? \\
- Have any of the symptoms listed above been worse after eating fatty foods? \\
\hline
\end{tabular}


frequency, bad odour, increased flatulence, abdominal cramps, bloating, and worsening of symptoms after eating fatty foods. Patients were asked to report any symptoms that had occurred on more than one occasion during the previous two weeks, irrespective of severity. Patients completed the questionnaire at the time of enrolment to the study and for a second time following treament with PERT $\left(\mathrm{Creon}^{\circledR}\right)$. The questionnaires were completed either at face-to-face interview with a researcher or by telephone follow-up. All patients had received PERT for at least 30 days prior to completing the second questionnaire.

\section{Assesmment of quality of life}

Quality of life was studied using the Short Form 36 (SF-36) health survey. The SF-36 is a patient reported measure of patient health and gives a breakdown of quality of life across nine separate physical and emotional health domains allowing specific health outcomes to be studied individually. The SF-36 questionnaire is internationally recognised and has been validated across numerous medical conditions making it an appropriate measure of quality of life in this setting (11). Patients completed the questionnaire at the time of enrolment to the study and following PERT and the survey was undertaken at the same time as completing the questionnaire relating to assessing symptoms of EPI.

\section{Measurement of faecal elastase}

Pancreatic exocrine function can be tested directly or indirectly. Direct tests make use of hormonal secretagogues of secretin and CCK to stimulate the pancreas and consequently the analysis of duodenal fluid for enzymes and bicarbonates. It is the most sensitive test for EPI. The indirect test may either be the measurement of faecal elastase-1, serum trypsinogen or faecal chymotrypsin. Faecal elastase- 1 is the most specific and sensitive of all three $(12,13)$. In this study, we measured faecal elastase- 1 as an indirect measure of pancreatic exocrine function. All samples were analysed by the biochemistry laboratory, Western General Hospital, Edinburgh. The normal range for faecal elastase- 1 is $>200 \mu \mathrm{g} / \mathrm{g}$. Values less than $200 \mu \mathrm{g} / \mathrm{g}$ were considered low and suggestive of severe EPI. We considered values between $200-500 \mu \mathrm{g} / \mathrm{g}$ to be borderline low.

At the start of the study we asked patients to provide a stool sample at the time of recruitment for measurement of faecal elastase. This was to determine if there was any association between faecal elastase concentration and patient-reported symptoms of EPI. Later in the study we stopped measuring faecal elastase and used the symptom questionnaire alone. This was because we noted in the interim analysis that all patients were reporting symptoms suggestive of EPI and it became clear that faecal elastase measurement was not adding meaningful additional information.

\section{Pancreatic enzyme replacement therapy (PERT)}

Creon ${ }^{\circledR}$ was chosen for pancreatic enzyme replacement. Patients identified with two or more symptoms suggestive of EPI from the responses to the questionnaire were offered PERT, irrespective of faecal elastase concentration (if measured). Patients were instructed to take 50000 units of Creon ${ }^{\circledR}$ with every meal and 25 000 units with every snack. Patients were informed that they would be contacted by telephone after several weeks of $\mathrm{Creon}^{\circledR}$ therapy to repeat both questionnaires (EPI questionnaire and SF-36)

\section{Statistical analysis}

No power calculation was performed as this study is considered a pilot study. The Wilcoxon matched pairs signed rank test and the Sign test were used for statistical analysis as the data was ordinal. The SF-36 output scores between 0-100 for each health domain; however, there are only a finite number of scores possible therefore the data is considered ordinal and was analysed accordingly. All statistical analysis was undertaken using SPSS and Minitab18.

\section{RESULTS}

\section{Study Population}

Sixty-seven patients were invited to take part in the study. Three patients subsequently withdrew and 7 patients were lost to follow-up before the questionnaires were completed therefore, 57 patients were recruited to the study. Patient demographics are shown in table 2. The median age was 68 years and 37 (65\%) patients were male. Thirty-six patients had previously undergone an esophagectomy and 21 patients had undergone either a subtotal or total gastrectomy. The average time from surgery to enrolment to the study was 686 days; the shortest interval between surgery and recruitment was 53 days and the longest interval was over 8 years. 
Table 2 - Study patient demographics $(n=57)$

( ${ }^{*}$ median; **mean)

\begin{tabular}{lc}
\hline Sex $(\mathrm{M}: \mathrm{F})$ & $37: 20$ \\
\hdashline Age (years) & $68^{*}($ range 50-84) \\
\hline Previous surgery & 36 \\
$\quad$ - oesophagectomy & 21 \\
$\quad$ - gastrectomy & $686^{\star *}$ \\
\hdashline Time interval between surgery and study recruitment & (range 53-2969) \\
\hline
\end{tabular}

\section{Assessment of symptoms of exocrine pancreatic insufficiency at study enrolment}

All 57 patients completed the questionnaire relating to symptoms of EPI (table 1). Only 1 patient reported no symptoms suggestive of EPI. The remaining 56 patients described at least two symptoms. Patient responses were scored 0 to 10 with the total score for each patient representing the number of positive symptoms. The most frequently recorded symptoms at recruitment were loose stools $(n=40(71 \%))$, foul smelling stools $(n=38(68 \%))$, bloating $(n=36(65 \%))$ and flatulence $(n=36(65 \%))$ (table 3). At the time of recruitment, the median abdominal symptom score reported by patients was 5 out of $10(\mathrm{IQR}=2$, range $0-10)$. There was no difference in symptom scores between patients who had an esophagectomy compared with gastrectomy, although the numbers for comparison are small. Likewise, there was no difference in symptom number and time interval from surgery.

\section{Faecal elastase concentration}

Faecal elastase concentration was measured for 24 patients. Twelve (50\%) patients had concentrations in excess of $500 \mu \mathrm{g} / \mathrm{g}, 10$ (42\%) patients had borderline

Table 3 - Patient responses to the abdominal symptom questionnaire before and after pancreatic enzyme replacement therapy (PERT) $(n=56)$

\begin{tabular}{lrr}
\hline Symptom & $\begin{array}{r}\text { Frequency of response } \\
\text { before PERT (\%) }\end{array}$ & $\begin{array}{r}\text { Frequency of response } \\
\text { after PERT (\%) }\end{array}$ \\
\hline Loose stool & $40(71 \%)$ & $7(13 \%)$ \\
\hline Foul odour & $38(68 \%)$ & $20(36 \%)$ \\
\hline Bloating & $36(65 \%)$ & $13(23 \%)$ \\
\hline Increased flatulence & $36(65 \%)$ & $27(48 \%)$ \\
\hline Increased frequency & $31(55 \%)$ & $9(16 \%)$ \\
\hline Difficulty flushing & $31(55 \%)$ & $16(29 \%)$ \\
\hline Cramps & $29(52 \%)$ & $16(29 \%)$ \\
\hline Worse after fatty food & $24(42 \%)$ & $4(7 \%)$ \\
\hline Weight loss & $18(32 \%)$ & $9(16 \%)$ \\
\hline Greasy stool & $18(32 \%)$ & $13(23 \%)$ \\
\hline
\end{tabular}

low levels of $200-500 \mu \mathrm{g} / \mathrm{g}$ and the remaining 2 (8\%) patients had low levels $<200 \mu \mathrm{g} / \mathrm{g}$. Patients $(n=10)$ with faecal elastase concentration less than $450 \mu \mathrm{g} / \mathrm{g}$ reported significantly more symptoms suggestive of EPI than those patients with faecal elastase concentrations greater than $450 \mu \mathrm{g} / \mathrm{g}$ : median 5 symptoms compared with 3 symptoms ((range $0-7$ symptoms), $p=0.043$; Mann Whitney U Test). However, all patients reported at least two symptoms suggestive of EPI irrespective of faecal elastase concentration.

\section{Assessment of symptoms of exocrine pancreatic insufficiency following PERT}

All patients who reported two or more symptoms suggestive of EPI were offered a trial of PERT (Creon ${ }^{\circledR}$ ). Therefore 56 out of the 57 patients were eligible for PERT. Patients were asked to repeat the abdominal symptom questionnaire following treatment with Creon $^{\circledR}$. The average duration of treatment prior to reassessment of symptoms was 88 days (range 31 - 199 days). Once again patient responses were scored 0 to 10 with the total score for each patient representing the number of positive symptoms. After introduction of PERT, the median symptom score reduced from 5 symptoms before treatment to 2 symptoms following treatment (IQR=2, range $0-5 ; Z=-4.734, p<0.0001$ Wilcoxon matched pairs signed ranks test) (fig. 1). The symptoms that patients reported had improved the most following PERT were loose stools (reduced from $71 \%$ of patients to $13 \%$ ) and increased stool frequency (reduced from $55 \%$ to $16 \%$ ) (table 3). One patient withdrew from the study due to vomiting, which may or may not have been related to Creon ingestion. Two patients reported new onset constipation following commencement of Creon but these patients continued taking Creon due to improvements in other symptoms.

\section{The effect of PERT on quality of life}

Patients were asked to complete the SF-36 quality of life questionnaire at the same time as completing the abdominal symptom questionnaire. Thirty-one patients completed both questionnaires before and after PERT. We selected 5 out of the 9 SF-36 health domains for analysis as they best represent the issues faced by our study population, namely; physical functioning, role limitations due to physical health, energy/fatigue, emotional well-being and social functioning. The SF-36 questionnaire generates scores between 0-100 $10=$ lowest possible QoL, 100 = highest possible QoL). PERT increased quality of life scores for every patient in 


\begin{abstract}
Figure 1 - Patient reported symptoms suggestive of exocrine pancreatic insufficiency (EPI) before (blue) and after (red) pancreatic enzyme replacement therapy (PERT). (a) Frequency of abdominal symptoms (b) Median total abdominal symptom score (Wilcoxon signed ranks test; $p<0.0001$ )
\end{abstract}

a Frequency of abdominal symptoms
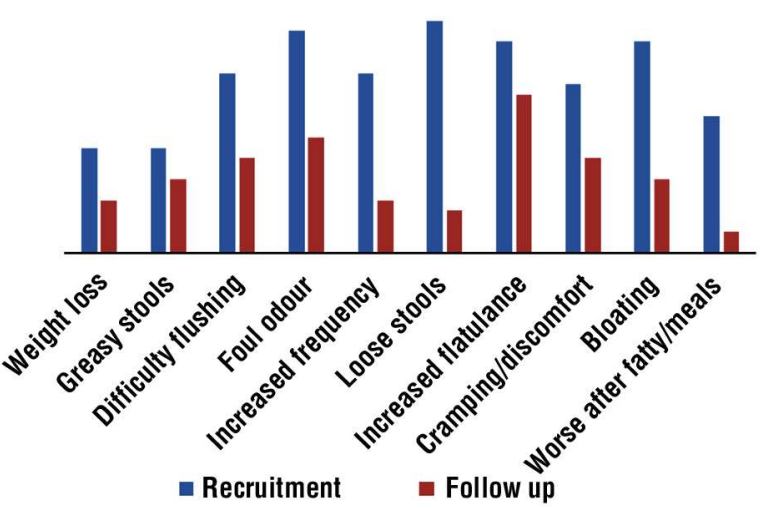

Abdominal symptom scores

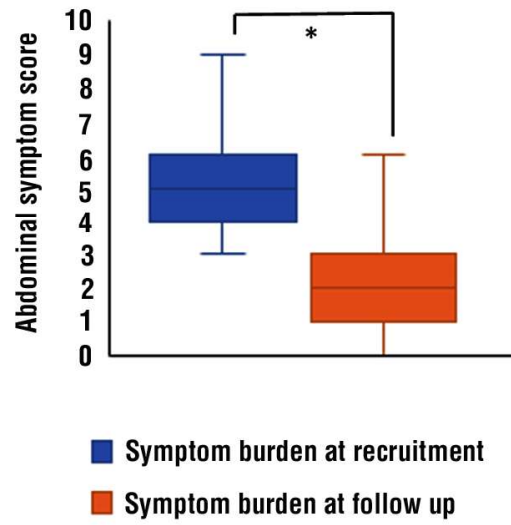

each of the 5 health domains (shown in fig. 2). Mean physical function score improved from 75.0 to 88.7 ( $Z=-4.118, p=0.0004$; Sign test). Mean role limitation due to physical health score improved from 42.7 to 67.7 ( $p=0.0005$, $z$ statistic not applicable as the $p$-value was generated using the exact method). Mean energy/ fatigue score improved from 50.2 to 63.5 ( $Z=-2.008$, $p=0.045)$. Mean emotional well-being score improved from 78.8 to 85.7 ( $Z=-2.600, p=0.009)$. Mean social functioning score improved from 78.2 to $88.7(p=0.008$, $\mathrm{z}$ statistic not applicable as the $\mathrm{p}$-value was generated using the exact method).

\section{DISCUSSION}

In this study almost every patient reported symptoms suggestive of exocrine pancreatic insufficiency following surgery for oesophageal or gastric cancer and every patient described some improvement in these unpleasant abdominal complaints following treatment with pancreatic enzyme supplementation (PERT). In addition, all patients reported significant improvement in their quality of life scores following PERT.

Our study population is typical of a patient cohort following upper gastrointestinal cancer surgery and

\title{
SF-36 scores at recruitment vs follow-up
}

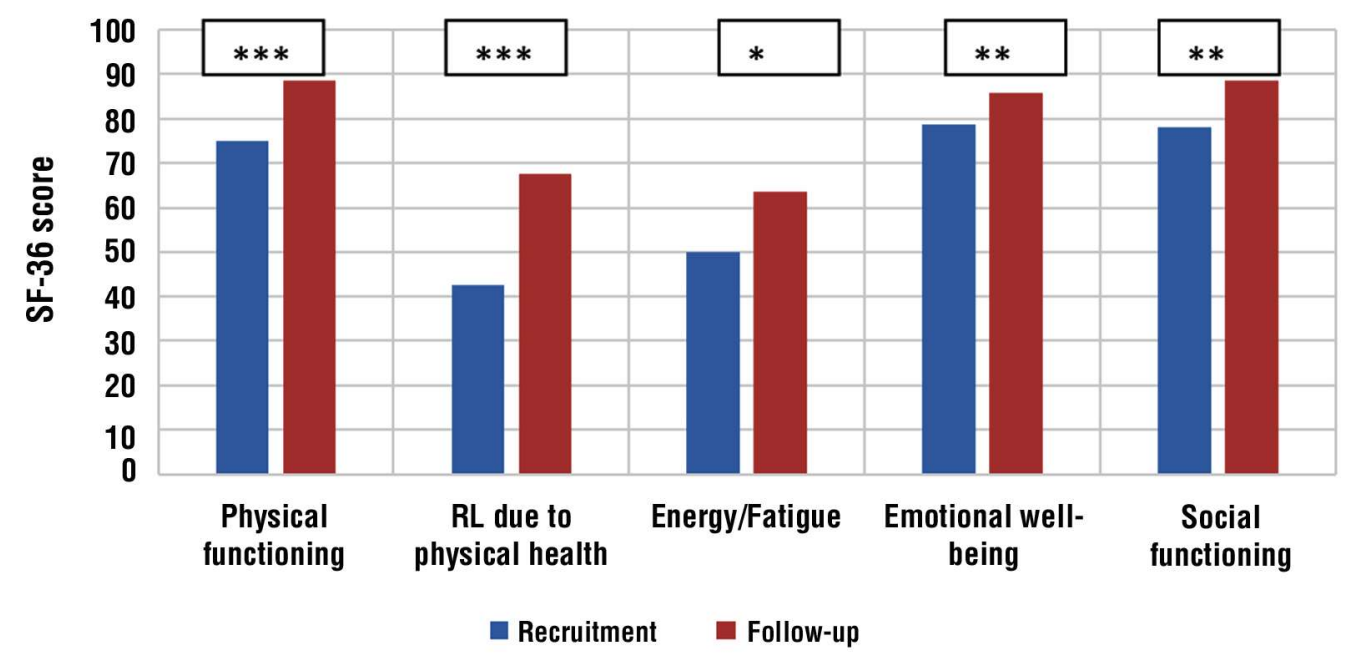

Figure 2 - Mean SF-36 QoL scores for five health domains comparing patient scores before (blue) and after (red) treatment with PERT (Sign test, ${ }^{*} \mathrm{p}<0.05,{ }^{* \star} \mathrm{p}<0.01,{ }^{* \star *} \mathrm{p}<0.001$ ) 
includes patients from a range of time intervals following surgery with an average interval of almost two years, but ranging from seven weeks to over eight years. Unpleasant abdominal symptoms are common among this patient group and are often regarded as an inevitable consequence of surgery. Moreover, these symptoms are often attributed to 'dumping syndrome' and other conditions which may lead to chronic maldigestion syndromes and are often regarded as untreatable (2). The present study has shown that this is not the case and that all patients with abdominal symptoms in-keeping with EPI will gain symptomatic benefit from PERT. Our results show a reduction from a median five abdominal complaints to two following PERT.

The current study has also provided a novel insight into the effect of PERT on quality of life. Using the SF-36 questionnaire we undertook a comprehensive investigation of global QoL across nine health domains. The most significant improvements were seen in the domains of physical function, social function and emotional well-being. Improvements in social function and emotional well-being can be easily linked with improvements reported in symptoms of unpredictable and unreliable bowel patterns which had limited social activities prior to PERT. With survivorship continually improving in oesophageal and gastric cancer it is becoming increasingly important to consider means of improving QoL for these patients.

Previous studies have described variable outcomes. A systematic review conducted in 2017 of EPI after gastrectomy identified only four suitable articles for analysis (4). This review found there was a significant decrease in exocrine pancreatic function following gastrectomy, but noted only minor improvement in symptoms following PERT. The authors did acknowledge that the review included poor quality studies involving small study numbers. A year later a randomised controlled trial was undertaken where patients were randomised to PERT or normal diet for 12 months following gastrectomy (14). This study reported increased serum pre-albumin concentration and higher Gastrolntestinal Quality of Life Index (GIQLI) scores in the treatment group, but these improvements were only noted for the first three months following surgery. Another study was published in the same year where PERT was prescribed to patients following esophagectomy who reported symptoms of steatorrhoea. When compared with patients without steatorrhoea and who did not receive PERT, the treatment group gained more weight during their recovery phase following surgery (15). We did not measure nutritional variables in the present study. Instead our aims were to investigate the effect of PERT on abdominal symptoms and quality of life. However, it is hypothesised that there will be nutritional benefits to PERT for patients with impaired intestinal digestion and absorption, especially for absorption of fat-soluble vitamins and other micronutrients.

We did measure faecal elastase concentration in a subgroup of our study patients. Previously, studies using faecal elastase as a marker of pancreatic exocrine function demonstrated that almost $80 \%$ of patients experience a degree of EPI following esophagectomy (3). A similar reduction in pancreatic exocrine function was reported in post-gastrectomy patients by Friess et al, using direct pancreatic stimulation techniques (secretin-cerulein test) to measure pancreatic function (6). Conversely Ward et al published data to suggest there was no reduction in exocrine pancreatic function following esophagogastric resection through use of carbon 13 labelled mixed triglyceride breath testing (C13-MTG-BT) (16). However, the authors highlighted that altered post-operative stomach transit time may have impacted on the validity of their methods.

In our study reduced faecal elastase concentrations were identified in half the patients that were tested. Only two patients had faecal concentrations below the normal range $(<200 \mu \mathrm{g} / \mathrm{g})$, however, it is important to note that all patients in the current study reported two or more symptoms suggestive of EPI despite a 'normal' faecal elastase concentration. This may be explained in several ways. It is possible that although production of enzymes from the pancreas is normal there may be disruption to the signaling pathways that initiate a coordinated secretion of these enzymes as a consequence of the surgery. As a result, there is a 'functional' exocrine deficiency and symptoms are improved due to Creon being administered at the time it is required. Alternatively, some of the symptoms reported by the patients are in fact not due to EPI at all but are related to other conditions such as dumping syndrome and small intestine bacterial overgrowth and the reported improvement in symptoms are a consequence of a placebo effect. A randomised controlled trial or cross-over study would be required to help clarify this explanation.

Other limitations of this study relate to the prescription of the pancreatic enzyme replacement. All patients were prescribed Creon and instructed to take 25000 units with snack and 50000 units with meals. It is possible that further improvements may be achieved by ensuring correct timing, dosing adjustments and compliance through specialist dietetic input. Creon is 
cheap, safe and well-tolerated by patients. Only one patient in our study stopped taking Creon due to adverse symptoms due to vomiting. Two other patients described new onset constipation, but continued to take Creon due to improvement in other abdominal symptoms.

Despite these limitations, this pilot study has identified that symptoms of EPI are common in patients following esophagogastric cancer surgery and these symptoms can be identified by a list of simple questions without the need for additional measurement of faecal elastase. This small study has demonstrated a proof of concept that PERT, such as Creon, reduces unpleasant abdominal symptoms and this leads to significant improvements in quality of life across five health domains. A randomised controlled trial should be considered as the next step to further investigate EPI among these patients where the condition is extremely under-appreciated and under-treated. In conclusion, PERT should be considered for all post-operative UGI cancer patients with symptoms suggestive of EPI.

\section{Conflicts of interest and source of funding}

The authors have no conflict of interest to declare. No additional funding was received.

\section{Author contribution statement}

Chris Deans was responsible for the study concept and design. Callum Alexander, Robert Kay and Sajid Waheed Rahman were responsible for data collection and undertaking the study protocol and statistical analysis. All authors were involved in data analysis and in the preparation of the manuscript.

\section{Ethical approval}

Ethical approval for this study was agreed in line with the Edinburgh University Medical School guidelines and the study was granted an exception from requiring formal ethical approval through the Lothian Regional Ethics Committee.

\section{REFERENCES}

1 Antonini F, Crippa S, Falconi M, Macarri G, Pezzilli R. Pancreatic enzyme replacement therapy after gastric resection: an update. Digestive and Liver Disease. 2018; 50(1):1-5.

2 Huddy JR, Macharg FMS, Lawn AM, Preston SR. Exocrine pancreatic insufficiency following esophagectomy. Dis of the Esophagus. 2013;26(6):594-59.

3 Heneghan HM, Zaborowski A, Fanning M, McHugh A, Doyle S, Moore J, et al. Prospective Study of Malabsorption and Malnutrition After Esophageal and Gastric Cancer Surgery. Ann Surg. 2015; 262(5):803-7.

4 Straatman J, Wiegel J, van der Wielen N, Jansma EP, Cuesta MA, van der Peet DL. Systematic review of exocrine pancreatic insufficiency after gastrectomy for cancer. Dig Surg 2017;34(5):364-370.

5 Davis JL, Ripley RT. Postgastrectomy syndromes and nutritional considerations following gastric surgery. Surgical Clinics 2017; 97(2):277-293

6 Friess H, Böhm J, Müller MW, Glasbrenner B, Riepl RL, Malfertheiner P, Büchler MW. Maldigestion after total gastrectomy is associated with pancreatic insufficiency. American Journal of Gastroenterology 1996;91(2):341-7

7 Löser C, Möllgaard A, Fölsch UR. Faecal elastase 1: a novel, highly sensitive, and specific tubeless pancreatic function test. Gut 1996; 39(4):580-6.

8 Gullo L, Ventrucci M, Tomassetti P, Migliori M, Pezzilli R. Fecal elastase 1 determination in chronic pancreatitis. Dig Dis Sci 1999; 44(1):210-3

9 CREON® - Pancreatic Enzyme Replacement Therapy For EPI. https://www.creon.com/aboutcreon. Accessed May 2020.

10 Friess $\mathrm{H}$, Michalski CW. Diagnosing exocrine pancreatic insufficiency after surgery: when and which patients to treat. HPB. 2009; $11: 7-10$.

11 Struyvenberg MR, Martin CR, Freedman SD. Practical guide to exocrine pancreatic insufficiency - Breaking the myths. BMC Med 2017;15(1):29

12 Ali S, Gagloo M, Dhar S. Revisiting the problem of pancreatic exocrine insufficiency in surgical patients. The Internet Journal of Surgery 2012; 28(2): http://ispub.com/lJS/28/2/13811.

13 Ware JE and Gandek B. Overview of the SF-36 Health Survey and the International Quality of Life Assessment Project. J Clin Epidemiol 1998:51(11):903-912

14 Catarci M, Berlanda M, Grassi GB, Masedu F, Guadagni S. Pancreatic enzyme supplementation after gastrectomy for gastric cancer: a randomized controlled trial. Gastric Cancer. 2018:21(3):542-551.

15 Kiefer T, Krahl D, Osthoff K, et al. Importance of pancreatic enzyme replacement therapy after surgery of cancer of the esophagus or the esophagogastric junction. Nutr Cancer. 2018;70(1):69-72.

16 Ward S, Cade R, Mackay S, Hassen S, Banting S, Gibson P. Pancreatic exocrine function after major upper gastrointestinal surgery measured with a carbon 13 mixed triglyceride breath test. Gut 2012; 61(Suppl 2):A370-A370. 